\title{
Automatic Constraint Detection for Layout Regularization
}

\author{
Haiyong Jiang, Liangliang Nan, Dong-Ming Yan, Weiming Dong, Xiaopeng Zhang, Peter Wonka
}

\begin{abstract}
In this paper, we address the problem of constraint detection for layout regularization. As layout we consider a set of two-dimensional elements where each element is represented by its bounding box. Layout regularization is important for digitizing plans or images, such as floor plans and facade images, and for the improvement of user created contents, such as architectural drawings and slide layouts. To regularize a layout, we aim to improve the input by detecting and subsequently enforcing alignment, size, and distance constraints between layout elements. Similar to previous work, we formulate the layout regularization as a quadratic programming problem. In addition, we propose a novel optimization algorithm to automatically detect constraints. In our results, we evaluate the proposed framework on a variety of input layouts from different applications, which demonstrates our method has superior performance to the state of the art.
\end{abstract}

Index Terms - layout regularization, constraint detection, constraint analysis, linear integer programming.

\section{INTRODUCTION}

$2 \quad$ We propose an algorithm for the regularization of lay3 outs. In this paper, a layout refers to a two-dimensional 4 arrangement of objects. Layouts arise in a variety of ap5 plications, for example they can come from digitized ar6 chitectural floor plans, digitized facade images, image and 7 text layouts on slides, line drawings, and graph drawings. 8 In practice, when a layout is designed or digitized from 9 another source (e.g., images), it is inevitable that noise oc10 curs through imprecise user input. Elements in an idealized ${ }_{11}$ layout exhibit some regularities, e.g., they are aligned, of 12 same-size, or uniformly distributed along a specific direc13 tion. However, in the aforementioned applications these 14 regularities typically disappear due to the approximate user 15 input. In this work, we want to detect and restore these 16 regularities, eliminating the noise that occurred during the 17 layout design or digitization stage.

18 We see three reasons why this is an important problem. ${ }_{19}$ First, high-level shape analysis is a popular topic in com2o puter graphics. Many available methods rely on correctly 21 extracted relationships to analyze a scene [1]. Even if the 22 input and output of our regularization look visually simi${ }_{23}$ lar, it is important that correct relationships are extracted. ${ }_{24}$ Our motivation for this paper, was to build datasets for

- H. Jiang is with the National Laboratory of Pattern Recognition (NLPR), Institute of Automation, Chinese Academy of Sciences, Beijing 100190, 31 China, and KAUST, Thuwal 23955-6900, Saudi Arabia. Email: haiyong.jiang@nlpr.ia.ac.cn.

- L. Nan is with KAUST, Thuwal 23955-6900, Saudi Arabia. Email: liangliang.nan@gmail.com

- D.-M Yan is with KAUST, Thuzval 23955-6900, Saudi Arabia, and the National Laboratory of Pattern Recognition (NLPR), Institute of 36 bottom aligned with another). 3) Elements can be missing Automation, Chinese Academy of Sciences, Beijing 100190, China. Email: ${ }_{37}$ from a regular pattern. 4) Spacing between rows or/and
yandongming@gmail.com. D.-M. Yan is the corresponding author.

- W. Dong and X. Zhang are with the National Laboratory of Pat- 38 columns can be irregular. Fig. 1 shows the complexity of tern Recognition (NLPR), Institute of Automation, Chinese Academy 39 possible constraints in an example layout. of Sciences, Beijing 100190, China. Email: weiming.dong@ia.ac.cn, xiaopeng.zhang@ia.ac.cn.

- P. Wonka is with KAUST, Thuwal 23955-6900, Saudi Arabia, and 41 layout model. A simple layout model has only a few paramArizona State University, Tempe, AZ 85287-8809. E-mail: pwonka@ 42 eters and therefore the fitting process is fairly robust. These gmail.com.

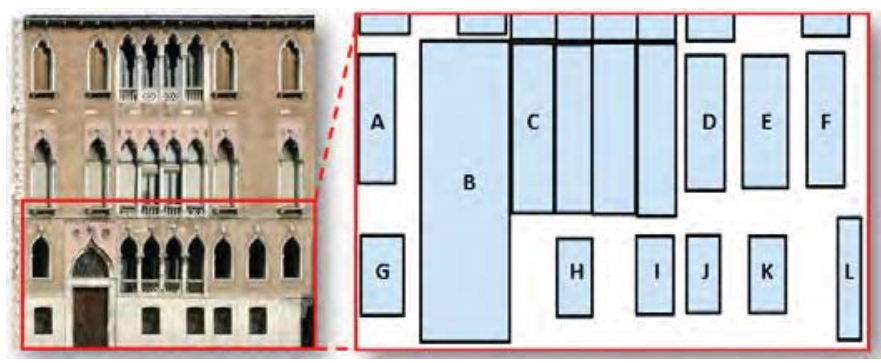

Fig. 1 Complexity and multiformity of constraints in a facade layout. Elements $B$ and $C$ are partially aligned (only top aligned, but not bottom aligned). The large element $B$ is aligned with different objects at the top (C ... F) and bottom (L). Elements are missing from a regular pattern consisting of A, D, E, and F. Spacing between same-sized elements $H, I, J, K$ is irregular.

machine learning techniques for layout synthesis. Second, a regularized layout compresses better than a noisy one. outs efficiently. Third, in most cases the visual difference 29 are noticeable and the regularized layout looks better than

Regularization of layouts is challenging in several ways 32 and we discuss a few selected example challenges: 1) Ele34 tom aligned, but not top aligned). 2) Large elements can be

A key ingredient in regularization is the design of the 43 simple models, e.g., a set of regular grids, are popular for the original one. зз ments can be partially aligned (e.g., elements are only bot- 
44 automatic pattern analysis in images [2] and 3D shapes [3],103 split grammar to parse the facade images. Teboul et al. [13] 45 [4]. Unfortunately, this simple data model limits the appli-104 parse facade layouts by using reinforcement learning. Wu et 46 cability to a large class of layouts, e.g., the layout shown in 105 al. [1] extract grammars from labeled facade layouts, and ${ }_{47}$ Fig. 1. A complex model typically has many parameters and ${ }_{106}$ generate large scale variations by editing the grammars. $48 \mathrm{can}$ fit a large number of layouts. However, the model is not ${ }_{107}$ Shen et al. [14] adaptively partition urban facades into a 49 very robust to noise.

108 hierarchical structure based on concatenated and interlaced 50 An initial framework for regularization was presented ${ }_{109}$ grids. Musialski et al. [15] developed an interactive tool for 51 by Pavlidis and Van Wyk [5]. They propose a simple greedy ${ }_{110}$ facade image segmentation, where a significant amount of 52 algorithm to detect constraints from a layout. By constrast, 111 user interaction is required. While most of these analysis of ${ }_{53}$ we use an optimization approach based on four steps.112 the facade layouts use a hierarchical representation, Zhang ${ }_{54}$ First, we extract constraint candidates. Second, we score the 113 et. al. [16] proposed to model layouts using layered grids 55 likelihood of constraints using energy functions. Third, we 114 with irregular spacing. In our work, we also use grids with 56 use global optimization using linear integer programming ${ }_{115}$ irregular spacing, but we can avoid the complexity of the 57 to select a subset of the constraint candidates that work well 116 layered structure.

58 together. Fourth, we regularize the layout by transforming ${ }_{117}$ Geometry structure analysis. In the 3D space, quite a few 59 the contents of the layout such that the change in both of ${ }_{118}$ papers focus on discovering regular patterns for geometry 60 the element locations and sizes is minimal while respect-119 structure analysis. Mitra et al. [17] propose a pair matching ${ }_{61}$ ing the selected constraints. Our formulation of the layout ${ }_{120}$ based approach to detect partial and approximate symmetry 62 regularization is an energy minimization using quadratic ${ }_{121}$ in $3 D$ shapes. Pauly et al. [18] further introduce a framework ${ }_{63}$ programming.

122 for detecting translational, scaling and rotational patterns 64 In our results, we will show that our algorithm has much ${ }_{123}$ in 3D shapes. Tevs et al. [19] build a connection among 65 better performance than [5] and also better performance ${ }_{124}$ similar shapes via geometric symmetries and regularities. 66 than the independently developed algorithm in [6]. Further, 125 These approaches have inspired many applications, such as 67 our framework has more types of constraints that can be 126 shape analysis, reconstruction, and synthesis. For example, 68 considered than previous work. The constraints we consider ${ }_{127} \mathrm{Li}$ et al. [20] propose to reconstruct 3D shapes from noisy 69 are constraints on the size, spacing, and alignment of layout ${ }_{128}$ and incomplete point cloud data that simultaneously de70 elements.

We make the following contributions: 129 tects surface primitives and their mutual relationships. This 130 approach involves both local and global shape analysis. We

- We propose a formulation of the layout regulariza- ${ }^{131}$ refer the reader to the recent survey paper of [21] for more tion problem that has better performance than previ- ${ }^{132}$ related work in this topic.

ous work, as evaluated on a test dataset consisting of ${ }^{133}$ Layout enhancement. The layout enhancement (regularlayouts from a variety of applications.

134 ization and beautification) has been studied in different 135 areas, e.g., object alignment [22], handwriting and drawing - We extend previous work by including a larger vari- ${ }^{-}$beautification [23], [24], [25], sketch and drawing beautifiety of constraints that can be detected and considered ${ }_{137}^{136}$ cation [5], [6], [26], [27], and 3D shape symmetrization [28]. in the layout optimization.

\section{Related Work}

${ }_{138}$ Nan et al. [29] exploit and model conjoining Gestalt rules 139 for facade elements grouping and summarization. AlHa140 lawani et al. [30] analysze and edit the facade images with ${ }_{80}$ The layout problem can be roughly classified into two major ${ }_{141}$ (semi-)regular grid structures. Huang et al. [31] combine ${ }_{81}$ categories, i.e., seamless layouts without gaps, and layouts ${ }_{142}$ patch-based image completion and translational symmetry 82 with gaps between elements. Our work focuses on the latter ${ }_{143}$ detection to fill the missing part of an incomplete planar 83 type of layout problems. We review the most related work in ${ }_{144}$ structure. More recently, $\mathrm{Xu}$ et al. [32] propose a command84 image structure analysis, geometry structure analysis, and ${ }_{145}$ based arrangement tool for 2D layouts.

85 layout enhancement.

146 Pavlidis and Van Wyk [5] beautify drawings using a clus${ }_{86}$ Image structure analysis. There is a large amount of struc- ${ }^{-147}$ tering method, while $\mathrm{Xu}$ et al. [6] interactively enhance the 87 ture analysis literature that addresses different aspects of ${ }_{148}$ global beautification with user guidance. We will compare 88 image analysis and understanding. A common interest in ${ }_{149}$ our approach to these two methods in Sec. 6.

89 both computer graphics and computer vision is the fa-150 In this work, we are interested in processing digitized 90 cade layout analysis for urban modeling [7]. The image ${ }_{151}$ 2D images and drawings. By abstracting each layout as a ${ }_{91}$ labeling problem has been addressed by considering both ${ }_{152}$ set of rectangles, our goal is to regularize the layout of the 92 visual evidence and architectural principles [8]. Based on ${ }_{153}$ elements such that the regularities of the elements in the 93 a perceptual grouping approach, translational symmetry is 154 layout are enforced.

94 exploited for single-view image recognition [9]. A similar 95 approach that uses the repetition information for facade

${ }_{96}$ image reconstruction is proposed by $\mathrm{Wu}$ et al. [10]. To ${ }^{155} 3$ OVERVIEW

97 understand the structure of a facade, a set of facade im-156 Given an image or drawing $\mathbf{I}$ that is characterized by a 98 ages are first recursively split and labeled for training, and ${ }_{157}$ set of rectangles, the layout $L=\left\{e_{1}, \ldots e_{n}\right\}$ of $\mathbf{I}$ can be 99 then the features are extracted from the segmented facades 158 simply described as the locations and sizes of the elements 100 and are used to guide the labeling. Riemenschneider et 159 in I. Here, an element $e_{i}$ is defined by a label $l_{i}$, and its 101 al. [11] combine both low-level and mid-level classifiers 160 bounding box $b_{i}=\left\{x_{i}, y_{i}, w_{i}, h_{i}\right\}$ depicting its bottom-left 102 for irregular facade parsing. Yang et al. [12] use a binary ${ }_{161}$ corner $\left(x_{i}, y_{i}\right)$ and the size $\left(w_{i}, h_{i}\right)$ (see Fig. 4). Our goal 


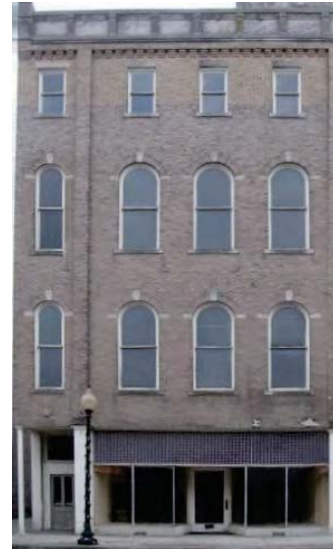

(a)

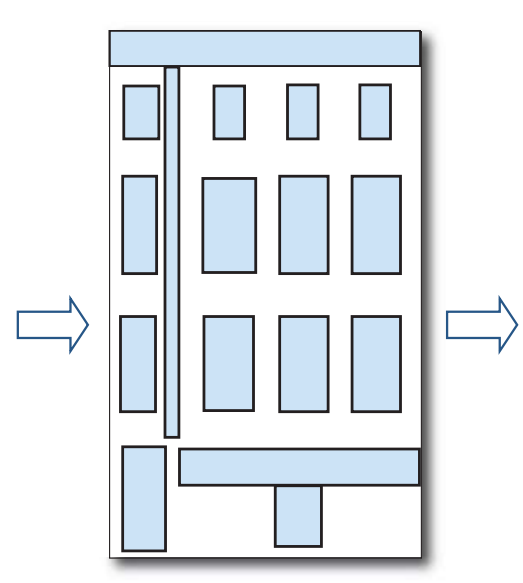

(b)

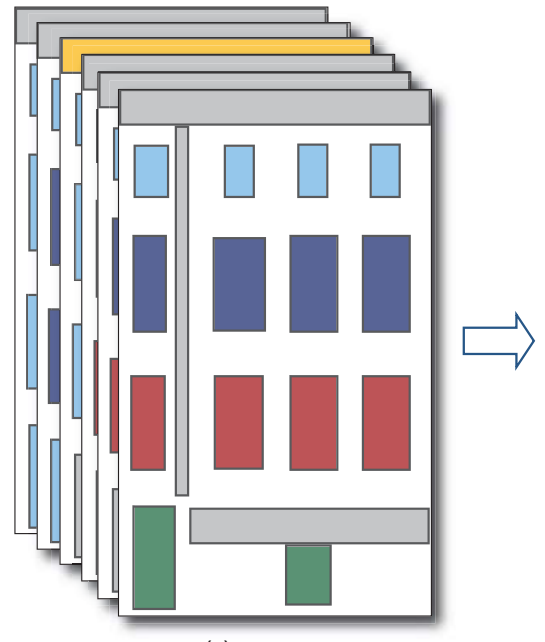

(c)

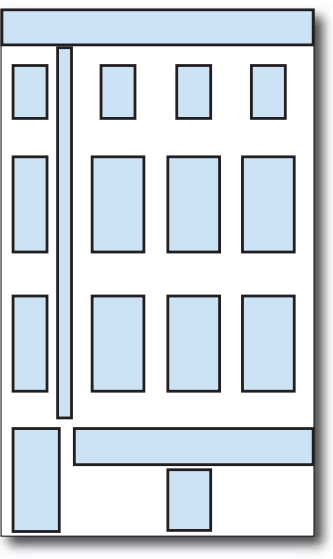

(d)

Fig. 2 An overview of our layout regularization approach. Given an input image or drawing (a), we first obtain the initial layout by manually marking and labeling the elements in a pre-processing step (b). Then appropriate constraints are automatically selected (c) and are used to generate the regularized layout (d).

162 is to regularize the layout of the elements such that the 163 regularities of these elements are enforced.

164 Our proposed solution to the layout regularization prob165 lem uses both discrete and continuous optimization. Fig. 2 166 shows an overview of our layout regularization method. ${ }_{167}$ Our method consists of the following three steps.

${ }_{168}$ Preprocessing. To digitize the layout of a given image, 169 the user manually marks and labels the elements in the 170 input image. The output of the preprocessing step is the 171 initial layout that will be regularized in the next steps. 172 Alternatively, the input can be user generated drawings or 173 slide layouts.

${ }_{174}$ Constraint selection. We first detect a larger set of candidate 175 constraints from the initial layout using a simple threshold176 ing based method. Then we score each constraint using an ${ }_{198}$ 177 energy function. Finally, we select a set of constraints from 178 the candidates using global optimization (linear integer
179 programming). Details on constraint selection are described ${ }_{180}$ in Section 4.

180 in Section 4.

${ }_{181}$ Layout regularization. To regularize the input layout, we 182 transform the contents of the layout such that the change 183 in both of the element locations and sizes is minimal while 184 respecting the selected constraints. We formulate the layout 185 regularization as energy minimization using quadratic pro186 gramming (Section 5).

\section{${ }_{187} 4$ Constraints Selection}

${ }_{188}$ Given the user marked elements in a layout, our layout 189 regularization tries to detect and enforce three types of 190 constraints: alignment, same-size, and same-spacing. This 191 problem is challenging in the following ways. First, we have 192 to detect reasonable constraints connecting elements in the 193 layout. Second, there may exist potential conflicts among 194 these constraints. To address these problems, we introduce 195 an optimization-based constraint selection algorithm. The 196 selected constraints are then used in a quadratic program197 ming formulation to regularize the layout (see Section 5).

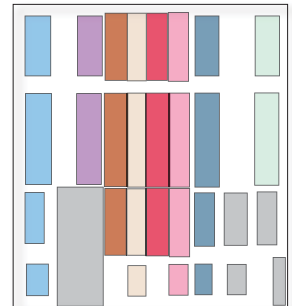

(a) Left alignment

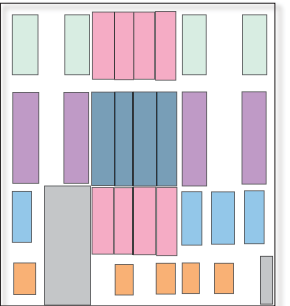

(b) Same size

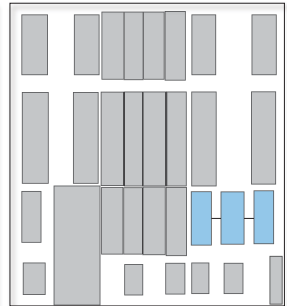

(c) Same horizontal spacing
Fig. 3 A subset of constraints in the example layout in Fig. 1. Colors indicate different constraint groups in this figure.

\subsection{Constraint Definitions}

99 We consider the following relationships between elements 200 as potential regularity constraints: alignment constraints, 201 same-size constraints, and same-spacing constraints (see Fig. 3 and Fig. 4).

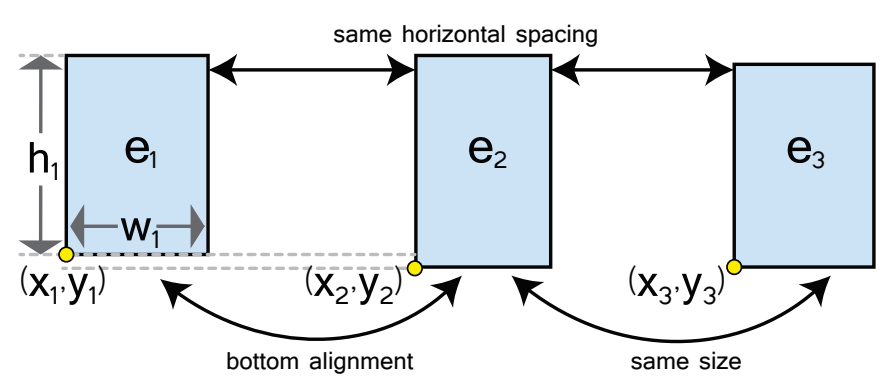

Fig. 4 Illustration of three types of constraints. The bottom alignment of element $e_{1}$ and $e_{2}$ can be formulated as $y_{1}-$ $y_{2}=0$. For the same size constraint of element pair $\left(e_{2}, e_{3}\right)$, we have $w_{2}-w_{3}=0$ and $h_{2}-h_{3}=0$. The horizontal same spacing constraint on element pairs $\left\{e_{1}, e_{2}\right\}$ and $\left\{e_{2}, e_{3}\right\}$ will turn out to be $x_{2}-\left(x_{1}+w_{1}\right)-\left(x_{3}-\left(x_{2}+w_{2}\right)\right)=0$. 
${ }_{203}$ Alignment constraints. Two elements $e_{i}$ and $e_{j}$ can have255 Same-spacing constraints. We take horizontal same-spacing 204 one or multiple of the following alignment constraints:256 as example. We sort all the element pairs in the input lay205 top alignment, middle-Y alignment, bottom alignment, left 257 out according to their horizontal intervals. Then the same206 alignment, middle- $X$ alignment, and right alignment. For 258 spacing constrained groups are generated by combining 207 example, a bottom alignment between $e_{i}$ and $e_{j}$ can be 259 element pairs so that each group satisfies the following two 208 formulated as

$$
y_{i}-y_{j}=0
$$

209 Other alignment relations are defined in a similar way. 260 conditions: 1) The difference in the interval of every element (1) ${ }^{261}$ pair is less than the threshold $t_{a} ; 2$ ) The elements overlap in 262 the vertical direction.

\section{${ }_{210}$ Same-size constraints. Two elements $e_{i}$ and $e_{j}$ may be 263 4.3 Energy Functions}

${ }_{211}$ linked by a same-width constraint or a same-height con- ${ }_{264}$ We now describe how to assign energy values to candidate 212 straint or both. Elements with the same label are always ${ }_{265}$ groups, so groups with lower energies are more likely to 213 considered to hold both same-size constraints. Same size $_{266}$ be selected. We first describe a set of auxiliary heuristic 214 constraints can be formulated as:

$$
\begin{aligned}
w_{i}-w_{j} & =0, \\
h_{i}-h_{j} & =0 .
\end{aligned}
$$
267 functions that will then be combined to obtain various 268 energy functions. In the optimization, we will use a linear (2) ${ }_{269}$ combination of the described energy functions as the objec270 tive function. A constraint group $g_{i}$ is composed of a set of ${ }_{215}$ Same-spacing constraints. Same-spacing constraints are de-271 elements $\left\{e_{1}, \ldots, e_{n}\right\}$ (2-element pairs for same-spacing). We 216 fined on 2-element pairs and can be either in the horizontal 272 define the following functions on $g_{i}$ :

217 or vertical direction. Currently, we only consider same-273 Standard deviation. The function stdvar $\left(g_{i}\right)$ measures the 218 spacing constraints between elements with the same labels.274 standard deviation of positions (for alignment constraints), ${ }_{219}$ For example, assume the element pairs $\left(e_{i}, e_{j}\right)$ and $\left(e_{m}, e_{n}\right) 275$ sizes (for same-size constraints), or spacings (for same220 should have the same spacing in the horizontal direction.276 spacing constraints). For example, if $g_{i}$ is a group of top${ }_{221}$ The equations for same-spacing constraints depend on the277 aligned elements, $\operatorname{stdvar}\left(g_{i}\right)$ is the standard deviation of 222 relative position of the elements. For the given examplezzs the top positions of all elements in group $g_{i}$.

223 assuming $x_{i}<x_{j}$ and $x_{m}<x_{n}$ would lead to

$$
x_{i}+w_{i}-x_{j}-\left(x_{m}+w_{m}-x_{n}\right)=0 .
$$

\section{4.2 Candidate Group Generation}

225 The candidate group generation step computes a set of ${ }_{285}$ for the scale of group $g_{i}$ in the relevant direction $(x$ or 226 candidate groups $\left\{g_{i}\right\}$, where each group $g_{i}$ is a set of el- ${ }_{286} y$ ). This function is evaluated differently for alignment, 227 ements that share an alignment, same-size, or same-spacing 287 same-size, and same-spacing constraints. For example, for 228 constraint. In this step, we use a threshold $t_{a}$ to limit the ${ }_{288}$ a group $g_{i}$ with horizontal alignment, $\operatorname{scale}\left(g_{i}\right)$ is equal to 229 candidate groups to a reasonably small set. Note that the 289 the minimal height of elements in group $g_{i}$. For same size 230 threshold $t_{a}$ is a global control mechanism for the number of ${ }_{290}$ constraints, scale $\left(g_{i}\right)$ is the maximum of the minimal width ${ }_{231}$ candidate groups being generated. This threshold is set high 291 and minimal height of the elements in group $g_{i}$. For same232 enough so that all reasonable candidates are generated. Note ${ }_{292}$ spacing constraints, we define $\operatorname{scale}\left(g_{i}\right)$ as the minimum ${ }_{233} t_{a}$ is only used for generating the candidate constraints, 293 spacing between element pairs in $g_{i}$.

234 while the actual constraints are selected using the linear in- ${ }_{294}$ In order to measure the quality of a constraint group, we 235 teger programming formulation described later. We describe 295 consider the following energy terms.

236 the candidate group generation for each constraint type $\mathrm{in}_{296}$ Intra-group distance. In our analysis, a good group should 237 the following.

297 have a small variance and a small maximal element dis${ }_{238}$ Alignment constraints. We use top-alignment as an exam-298 tance. Further, these values should be normalized by scale: 239 ple. We sort all the elements in the input layout according 240 to the $y$ value of their top edge. Let $\left\{p_{1}, \ldots p_{n}\right\}$ denote the 241 top positions of the sorted elements. We generate a set of

$$
E_{d}\left(g_{i}\right)=\frac{\max \left(0, \operatorname{stdvar}\left(g_{i}\right)+\max \operatorname{Dist}\left(g_{i}\right)-\epsilon\right)}{\operatorname{scale}\left(g_{i}\right)},
$$

${ }_{242}$ potential groups, such that the difference in the top positions 299 where $\epsilon$ is the maximal allowed tolerance value so that ${ }_{243}$ of every pair of the elements in each group are less than the 300 distances smaller than $\epsilon$ will be ignored. We set $\epsilon$ to 3 pixels 244 threshold $t_{a}$.

301 based on our experiments.

${ }_{245}$ Same-size constraints. For same size constraints, we first ${ }^{302}$ Aspect ratio variance. For same size constraints, the aspect 246 group all elements according to their label because we ${ }^{303}$ ratio plays an important role. Thus, we use an energy term 247 assume that elements with the same label have the same ${ }^{304} E_{a}\left(g_{i}\right)$ that captures the standard deviation of the aspect 248 size. Then, we compute the average element size for each ${ }^{305}$ ratio of all elements in group $g_{i}$. Here the aspect ratio of 249 label and use this element size to define a distance between 306 an element is defined as $\frac{w}{h}$, where $w, h$ are the width and 250 labels using the $l_{1}$ norm. For each label we find the k-nearest 307 height of the element.

251 neighboring labels, where $k$ is iterating from 1 to the number 252 of labels. This yields an initial set of candidate groups. Then ${ }^{308} 4.4$ Constraint Selection

253 we filter out candidate groups in which there exists two 309 We employ linear integer programming to select a set of ${ }_{254}$ elements with a size difference larger than the threshold $t_{a} .{ }_{310}$ constraint groups among the candidate groups. There are 
311 multiple goals: First, the energy values of the selected ${ }_{35}$

The optimization problem above is a linear integer pro${ }_{312}$ groups should be low. Second, the complexity of the overall ${ }_{352}$ gram that can be efficiently solved using various open 313 model measured by the number of constraint groups used 353 source solvers, e.g., [33], [34], [35]. The solution is a set 314 should also be low. This motivates the use of an additional 354 of constraint groups. Each group gives rise to a set of linear 315 sparsity term. In our formulation, each constraint type uses 355 equations that serve as constraints during the layout regu316 a different energy function.

356 larization step. For example, for an alignment group $g_{i}=$ 317 Given an input layout $L$ consisting of $n$ elements, and ${ }_{357}\left\{e_{i 1}, \ldots, e_{i N}\right\}$, we combine adjacent elements to form the 318 the candidate constraint groups $G=\left\{g_{1}, \ldots, g_{N}\right\}$ generated 358 constraint pairs, namely, $\left(e_{i 1}, e_{i 2}\right), \ldots,\left(e_{i(n-1)}, e_{i N}\right)$. Then 319 from $L$, our task is to choose a subset of these candidate 359 we generate one linear equation per constraint pair.

320 groups as constraints for the following layout regularization 321 step. Let $C=C_{a} \cup C_{s s} \bigcup C_{s p}$ denote all the constraint 322 types, where $C_{a}, C_{s s}$, and $C_{s p}$ are alignment, same size, and 323 same-spacing types, respectively. $\mathbf{Z}=\left\{z_{1}, \ldots z_{N}\right\}$ denotes 324 the binary label for each candidate group ( 1 for chosen and 0 325 for not chosen). We split $\mathbf{Z}$ into three subvectors $\mathbf{Z}_{\mathbf{a}}, \mathbf{Z}_{\mathbf{s s}}$, and ${ }_{326} \mathbf{Z}_{\mathrm{sp}}$ representing the labels for each type of the constraint ${ }^{34}$ ${ }_{327}$ groups. Then the energy for these types of constraint groups 328 are defined as follows:

329 Alignment constraints.

$$
E\left(\mathbf{Z}_{\mathbf{a}}\right)=\sum_{c_{j} \in C_{a}} \sum_{g_{i} \in G} E_{d}\left(g_{i}\right) \cdot z_{i} \cdot \delta\left(g_{i}, c_{j}\right)+w_{a} \cdot\left\|\mathbf{Z}_{\mathbf{a}}\right\|_{0},
$$

${ }_{330}$ where $\|\cdot\|_{0}$ denotes the $\ell^{0}$-norm, which counts the number ${ }_{372}$ regularized layout.

\section{LAYOUt REgularization}

With the optimal constraints detected and filtered from the 362 constraint selection step, our final goal is to regularize the 363 layout under these constraints. Our regularization process ${ }^{364}$ has a similar format with the methods of [36] and [37]. 365 These works both emphasize on the facade structure us366 ing a hierarchical layout, while ours deals with a layout 367 of rectangles. We address this regularization problem by 368 transforming the contents of the layout from the input 369 layout $L$ to regularized layout $L^{*}$ such that the change to 370 element locations $C_{L}$ and element sizes $C_{S}$ is minimal while 371 respecting the constraints. The star $\left(*\right.$ in $\left.L^{*}\right)$ indicates the ${ }_{331}$ of nonzero entries in a vector. We add this term to encour- ${ }_{373}$ To facilitate user preferences, we use a weight $\omega$ (we set 332 age fewer and larger groups (i.e., groups that have more 374 as 2.5 for our preference for position changes) to balance beззз elements). Since $z_{i} \in\{0,1\}$ in our problem, $\|\cdot\|_{0}$ can be 375 tween the two terms above. Then the layout regularization 334 simplified to the sum of all the entries in the vector. $\delta\left(g_{i}, c_{j}\right)_{376}$ is formulated as energy minimization as below:

335 is an indicator function that has value 1 if $g_{i}$ is a candidate 336 group of constraint type $c_{j}$, otherwise zero. $w_{a}$ is a weight 337 that balances the two terms.

${ }_{338}$ Same size constraints. The energy function for same size 339 constraints is similar to that of alignment constraints. To 340 account for aspect ratio of an element in the layout, we also ${ }_{341}$ involve the aspect ratio variance $E_{a}$ into the formulation:

$$
\begin{gathered}
E\left(\mathbf{Z}_{\mathbf{s s}}\right)=\sum_{c_{j} \in C_{s s}} \sum_{g_{i} \in G}\left(E_{d}\left(g_{i}\right)+w_{a} \cdot E_{a}\left(g_{i}\right)\right) \cdot z_{i} \cdot \delta\left(g_{i}, c_{j}\right) \\
+w_{s s} \cdot\left\|\mathbf{Z}_{\mathbf{s s}}\right\|_{0},
\end{gathered}
$$

$$
L^{*}=\arg \min \left(C_{L}+\omega \cdot C_{S}\right),
$$

where

$$
\begin{aligned}
& C_{L}=\sum_{i=1}^{n}\left(x_{i}^{*}+\frac{w_{i}^{*}}{2}-x_{i}-\frac{w_{i}}{2}\right)^{2}+\left(y_{i}^{*}+\frac{h_{i}^{*}}{2}-y_{i}-\frac{h_{i}}{2}\right)^{2} \\
& C_{S}=\sum_{i=1}^{n}\left(w_{i}^{*}-w_{i}\right)^{2}+\left(h_{i}^{*}-h_{i}\right)^{2}
\end{aligned}
$$

In addition to the aforementioned constraints selected in 378 Section 4, we add additional constraints to Equation 9 to (6) 379 ensure the validity of the optimized layout. In our formula${ }_{342}$ Same spacing constraints. For same-spacing constraints, the 380 tion, we include lower bound constraints and upper bound 343 energy function is similar to that of alignment constraints: 381 constraints for the variables, and sequential constraints for

$$
E\left(\mathbf{Z}_{\mathbf{s p}}\right)=\sum_{c_{j} \in C_{s p}} \sum_{g_{i} \in G} E_{d}\left(g_{i}\right) \cdot z_{i} \cdot \delta\left(g_{i}, c_{j}\right)+w_{s p} \cdot\left\|\mathbf{Z}_{\mathbf{s p}}\right\|_{0}{ }_{383}^{382}
$$
344 Afterwards, proper constraint groups are selected by ${ }^{385}$ 345 minimizing the following constrained objective function:

$$
\begin{array}{cll}
\underset{\mathbf{X}}{\operatorname{minimize}} & E\left(\mathbf{Z}_{\mathbf{a}}\right)+E\left(\mathbf{Z}_{\mathbf{s s}}\right)+E\left(\mathbf{Z}_{\mathbf{s p}}\right) & \\
\text { subject to } & \sum_{i=1}^{N}\left\|g_{i}\right\| \cdot z_{i} \cdot \delta\left(g_{i}, c_{j}\right)=n, & c_{j} \in C \\
& z_{i}+z_{j} \leq 1, \quad \forall g_{i} \bigcap g_{j} \neq \emptyset, & 1 \leq i, j \leq N \\
& z_{i} \in\{0,1\}, & 1 \leq i \leq N,
\end{array}
$$

346 where the constraints $\sum_{i=1}^{N}\left\|g_{i}\right\| \cdot z_{i} \cdot \delta\left(g_{i}, c_{j}\right)=n$ ensure 396 347 that every element in the layout is assigned to a constraint ${ }_{397}$ 348 group of type $c_{j}$. The second group of constraints $z_{i}+z_{j} \leq 1_{398}$ 349 enforce that groups do not have overlapping elements if $g_{i} 399$ 350 and $g_{j}$ are of the same constraint type. the elements' relative positions.

- Lower and upper bound constraints. These constraints restrict the changes of elements in reasonable ranges. Let $\left(w_{b}, h_{b}\right)$ denote the size of the bounding box of the layout, we add additional positional constraints $0 \leq x_{i}^{*} \leq w_{b}$ and $0 \leq y_{i}^{*} \leq h_{b}$.

Further, to prevent the elements from being changed too much in their sizes, we also add upper bound constraints on their sizes. Let's take the width bound as an example, it is defined proportionally to the widths of all elements that have the same label $\ell$. In our implementation, the maximal allowed width change for an element $e_{i}$ is defined as $\max (0.5$. $\left.\Delta w_{\ell}, 0.15 \cdot w_{i}\right)$, where $\Delta w_{\ell}$ is the maximal difference in width for elements that have the same label, and $w_{i}$ is the width of $e_{i}$. The size constraints on element height are defined similarly.

- Sequential constraints. These constraints specify the relative positions of pairs of elements. With these 
By solving the quadratic programming problem defined 409 in Equation 9, we obtain the regularized layout. In our 410 implementation, we add the constraints sequentially in 411 order to avoid potential conflicts. If there is any conflicts 412 detected during the optimization, we just remove the cur413 rent constraint. However, the sequence of constraints will 448 aligned. Then we have $\operatorname{num}(D)=4-1, \operatorname{num}(G)=5-1$, 414 affect the results. To incorporate our preferences for different 449 and $\operatorname{num}(G \cap D)=4-1$.

415 constraints, we sort all constraints according to their energy ${ }_{450}$ Comparison. We conducted comparisons with the methods 416 function stdvar $\left(g_{i}\right)$ (see Eq. 4), and then add them to the ${ }_{451}$ of $\mathrm{Xu}$ et al. [6] and Pavlidis et al. [5]. Pavlidis et al. [5] pro417 constraint set according to this order.

\section{${ }_{418} 6$ Results AND Discussion}

${ }_{419}$ Test database. Our experiments are conducted on a database ${ }^{456}$ the precision, recall, and F-measure for every layout in our 420 of 32 digitized layouts from various applications. Our data ${ }^{457}$ test database. As we can see, our algorithm has similar pre${ }_{421}$ set contains examples covering facades, slide designs, web ${ }^{458}$ cision to previous work, but higher recall for most layouts. ${ }_{422}$ designs, indoor scenes, and other graphical layouts. In Fig-459 This leads to the highest F-measure results on $93.8 \%$ of 423 ures 5, 6, and 7 we show a set of different layouts regu- ${ }^{460}$ layouts in the database. The comparison is also summarized 424 larized using our method. In the supplemental materials, ${ }^{461}$ in Tab. 1 left. In the next test, we compare the same-spacing 425 we provide more results showing detected relations and ${ }^{462}$ constraints. In Tab. 1 right, we show the comparison of the 426 regularized layouts. From these applications, we can see ${ }^{463}$ average values for precision, recall and F-measure. From ${ }_{427}$ that our method enforces the regularity constraints, while ${ }^{464}$ this comparison we see that the same-spacing constraint ${ }_{428}$ preserving the high-level relations, such as symmetries and ${ }^{465}$ is more difficult to detect than the alignment constraint. 429 the repetitive patterns.

466 Additionally, it was also very difficult to define a ground ${ }_{430}$ Evaluation metrics. In order to evaluate the effectiveness ${ }^{467}$ truth for this constraint. From the above comparison, we can ${ }_{431}$ of our framework, we designed an interactive program to ${ }^{468}$ see that the precision of the same spacing detection benefits ${ }_{432}$ specify the ground truth relationships for each layout. We ${ }^{469}$ from the label information. However our method still works ${ }_{433}$ use the marked relations to compute precision $(\mathrm{P})$, recall ${ }^{470}$ better than others even without the label information. In $434(\mathrm{R})$, and F-measure(F) defined as follows:

$$
\begin{aligned}
& P=\frac{\sum_{i \in G_{g}} \sum_{j \in G_{d}} \operatorname{num}\left(g_{i} \bigcap g_{j}\right)}{\sum_{j \in G_{d}} \operatorname{num}\left(g_{j}\right)}, \\
& R=\frac{\sum_{i \in G_{g}} \sum_{j \in G_{d}} \operatorname{num}\left(g_{i} \bigcap g_{j}\right)}{\sum_{i \in G_{g}} \operatorname{num}\left(g_{i}\right)}, \\
& F=\frac{2 \cdot P \cdot R}{P+R},
\end{aligned}
$$

${ }_{471}$ Fig. 9, we also show an illustrative example of a case where 472 our method is more successful than $\mathrm{Xu}$ et al. [6]. We can 473 see that $\mathrm{Xu}$ et al. can not handle layouts where elements 474 overlap with each other, thus it cannot align the elements 475 properly. In addition, we compare the performance of these 476 three methods by measuring the average computation time (10) ${ }^{477}$ for the examples in our dataset. The method in [5] could 478 detect the constraints in $0.001 \mathrm{~s}$ because of the simplicity of 479 the method. The method of $\mathrm{Xu}$ et al. [6] needs $0.898 \mathrm{~s}$, while 480 ours needs about $0.914 \mathrm{~s}$.

${ }_{481}$ Running time. We have implemented the proposed method 482 in $\mathrm{C}++$. All the experiments are performed on a PC with 435 where $G_{g}$ is the set of constraint groups in the ground truth, 483 two $2.70 \mathrm{GHz}$ Intel Xeon E5-2680 processors. We found that ${ }_{436} G_{d}$ is the set of constraint groups in the detected result, and ${ }^{484}$ the running time highly depends on the number of elements ${ }_{437} \operatorname{num}(\cdot)$ is the number of constraints in a constraint group. 485 and the number of relations in the input layout. On average, ${ }_{438}$ The term $g_{i} \cap g_{j}$ denotes the intersection of two constraint 486 the constraint selection step takes 0.70 seconds, and the ${ }_{439}$ groups. It is empty if $g_{i}$ and $g_{j}$ are of different types of con-487 regularization step takes 3.59 seconds. The maximum times 440 straints. For alignment and same size, an $n$-element con-488 were 3.71 seconds and 15.78 seconds, respectively.

${ }_{441}$ straint group will contribute $n-1$ constraint pairs, while an ${ }_{489}$ Robustness \& scalability. We evaluated the robustness and $442 n$-pair spacing group will contribute $n-1$ constraint pairs 490 scalability of our algorithm on synthesized examples. We ${ }_{443}$ (see Section. 4.1). Thus, we define the number of constraints 491 first generate a regular grid of elements of two different sizes 444 of a constraint group as the number of constraint pairs it ${ }_{492}$ with 8 columns and 5 rows. We then perturb the corners of 445 yields. For example, consider we have the top alignment of ${ }_{493}$ the elements with an increasing amount of Gaussian noise ${ }_{446}$ elements $G=\left\{e_{1}, e_{2}, e_{3}, e_{4}, e_{5}\right\}$ as ground truth, but the 494 (measured relative to the element sizes). The performance 447 algorithm only detects elements $D=\left\{e_{1}, e_{2}, e_{3}, e_{5}\right\}$ as top 495 of our method is demonstrated in Tab. 2. We can see that 


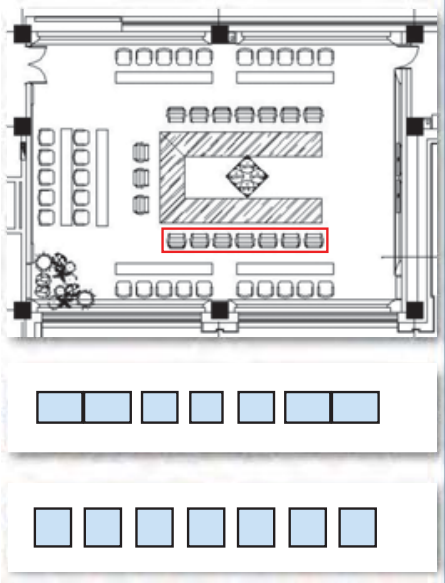

(a)

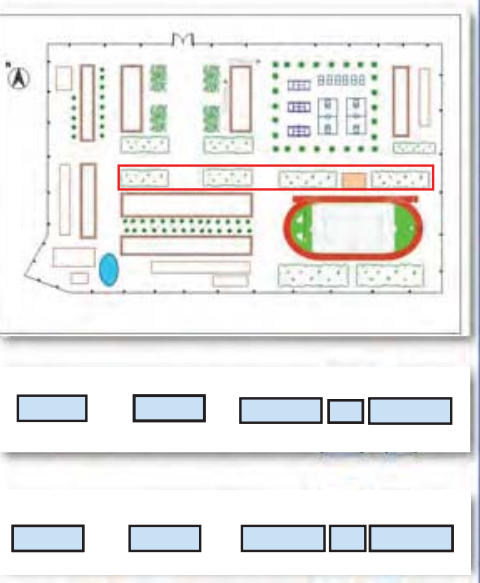

(b)

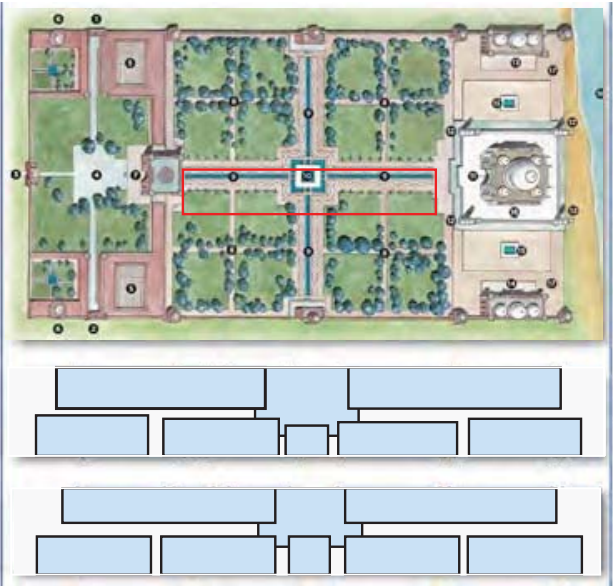

(c)

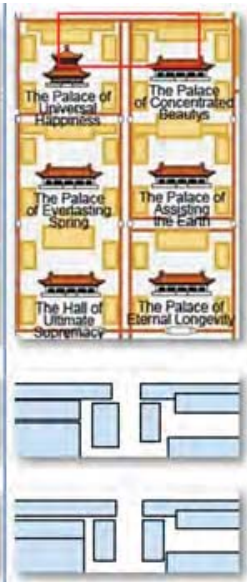

(d)

Fig. 5 Four different layouts are regularized using our method. Each column (from top to bottom) shows the input floor plan, zoomin of the marked region in the initial layout, and our regularized layout.

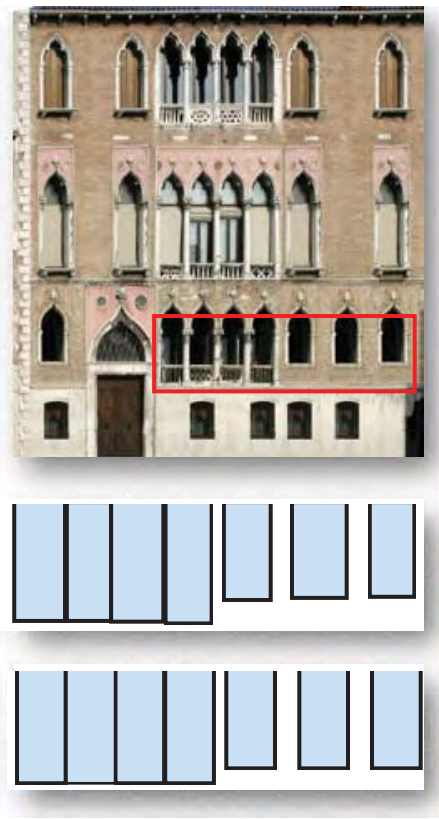

(a)

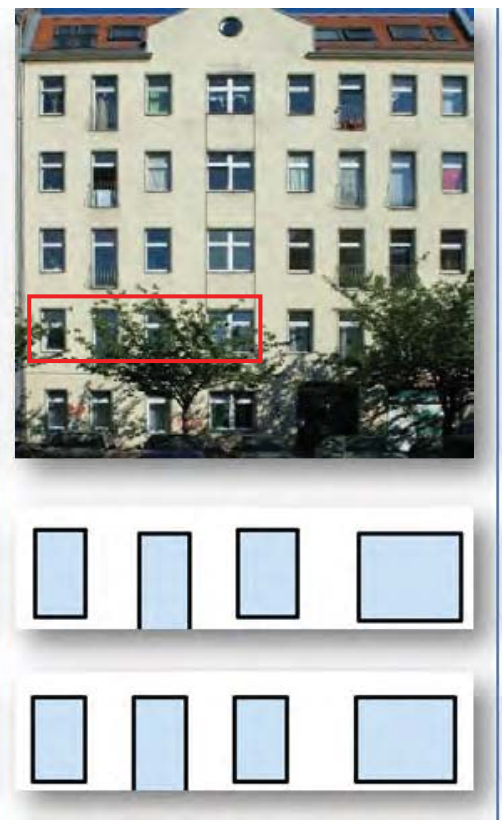

(b)

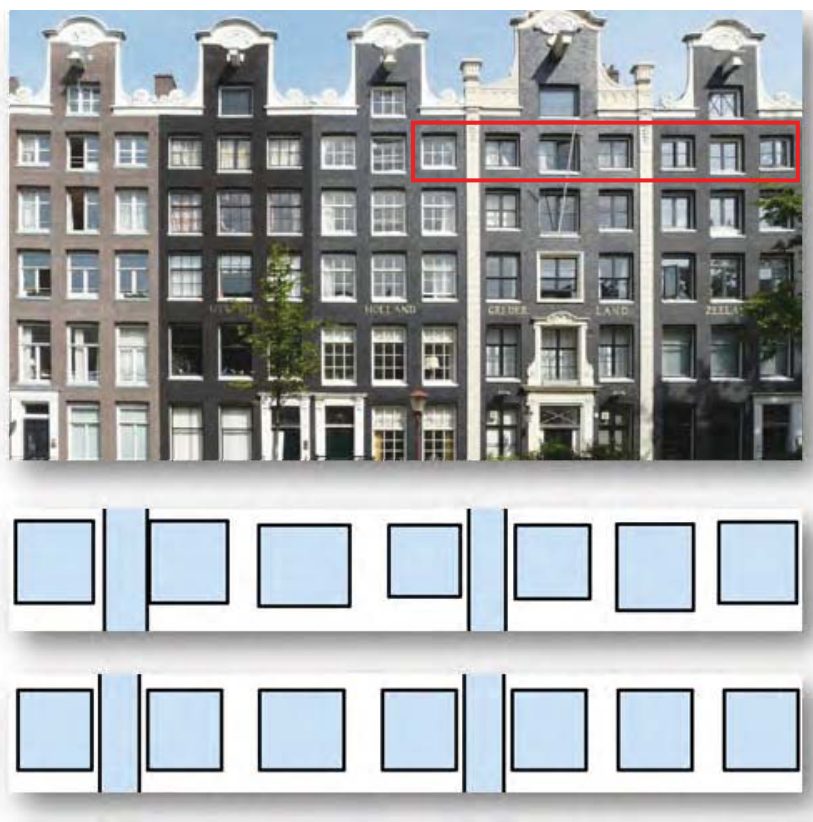

(c)

Fig. 6 Layouts regularization for a set of urban facade images. The top row shows the input facade images. The middle and the bottom rows show the zoomin views of the highlighted regions and the regularized results with abstract boxes.

496 our method works well if the noise is less than $10 \%$ of the 497 element size. To evaluate the scalability, we use Gaussian 498 noise with variance 0.02 and measure the running time for 499 grids with a different number of elements. In Tab. 3 we show 500 the results of this test. We can see that the accuracy decreases 501 with larger grids. The reason is mainly that some of the same 502 spacing constraints are not detected due to outliers.

503 Parameters. In our method, there exists multiple param504 eters. One parameter is the threshold $t_{a}$ that is used to 505 generate candidate groups. In order to verify the influence 506 of this parameter on the results, we evaluate our method 507 with different values of the threshold $t_{a}$ on the alignment 508 constraints (see Fig. 10). Our method can generate high 510 size, which makes our method reliable even without user 509 quality results after a value of 0.2 times the average element 511 intervention. Another important parameter is the weight for

TABLE 2 Performance of our algorithm on a data set with increasing amount of Gaussian noise relative to the element size. \#C is the number of detected constraints.

\begin{tabular}{|c|c|c|c|c|}
\hline Level of noise & \#C & P & R & $\mathrm{F}$ \\
\hline 0.00 & 321 & 1.000 & 1.000 & 1.000 \\
\hline 0.02 & 321 & 1.000 & 1.000 & 1.000 \\
\hline 0.04 & 319 & 1.000 & 0.993 & 0.996 \\
\hline 0.06 & 297 & 1.000 & 0.915 & 0.956 \\
\hline 0.08 & 290 & 1.000 & 0.894 & 0.944 \\
\hline 0.10 & 263 & 1.000 & 0.795 & 0.886 \\
\hline
\end{tabular}


This article has been accepted for publication in a future issue of this journal, but has not been fully edited. Content may change prior to final publication. Citation information: DOI 10.1109/TVCG.2015.2480059, IEEE Transactions on Visualization and Computer Graphics

JOURNAL OF TVCG

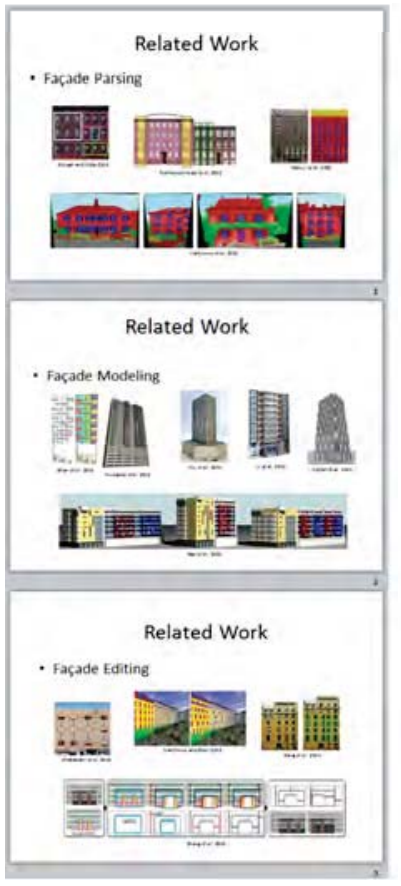

(a) Initial design

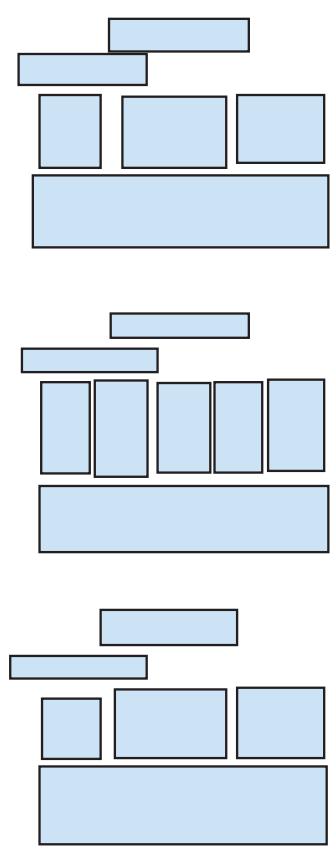

(b) Initial layout

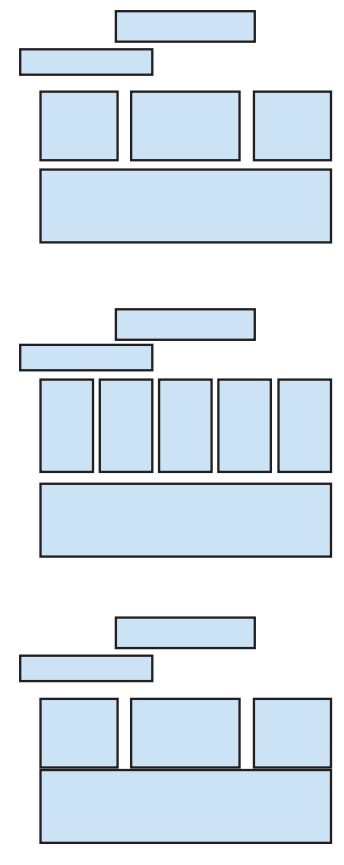

(c) Regularized layout

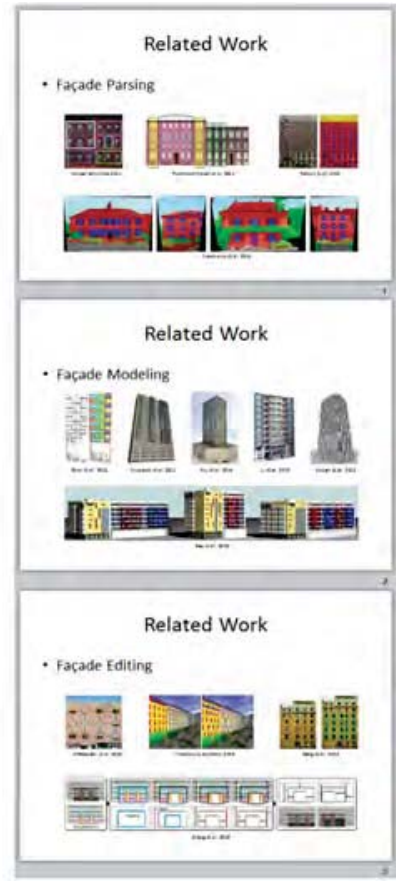

(d) Regularized design

Fig. 7 Slide design beautified using our approach. From left to right: the initial design, bounding boxes of the elements in the design as input layout, optimized layout, and the final design.
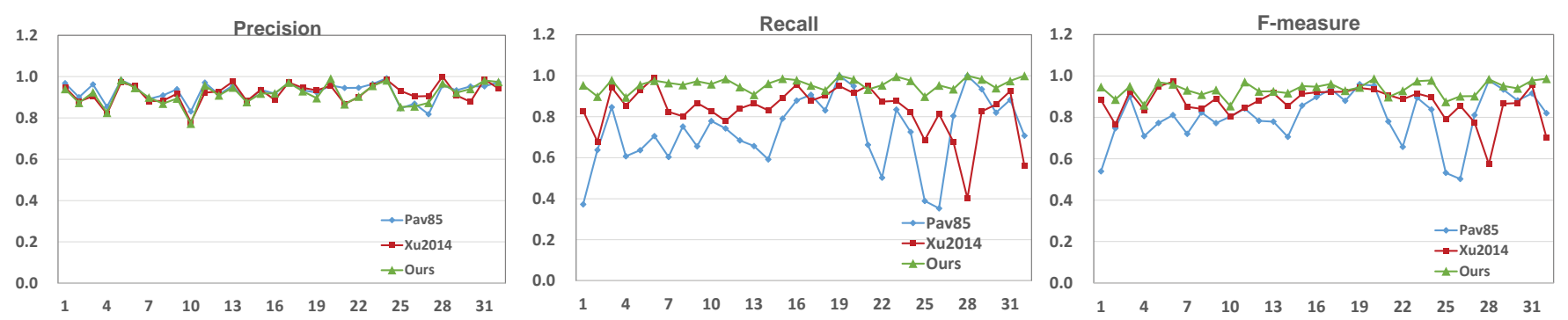

Fig. 8 The comparison of precision (left), recall (middle), and F-measure (right) of our method with Pav85 [5] and Xu2014 [6] on the alignment constraints.

TABLE 3 Performance of our algorithm on a data set with an increasing of number of rows and columns. Note that all the grid elements are perturbed by $2 \%$ Gaussian noise (relative to the element sizes).

\begin{tabular}{|c|c|c|c|c|c|}
\hline Grid size & \#C & P & R & F & Time(s) \\
\hline $5 \times 8$ & 321 & 1 & 1 & 1 & 2.245 \\
\hline $10 \times 8$ & 678 & 0.987 & 0.983 & 0.985 & 6.428 \\
\hline $5 \times 16$ & 676 & 0.975 & 0.986 & 0.981 & 6.996 \\
\hline $10 \times 16$ & 1420 & 0.964 & 0.971 & 0.967 & 25.789 \\
\hline $20 \times 16$ & 2940 & 0.947 & 0.964 & 0.955 & 121.822 \\
\hline
\end{tabular}

512 the sparsity term. Here, we evaluate the performance with 513 respect to the sparsity term $w_{a}$ as shown in Fig. 11. The 514 sparsity term plays an important role for selecting a trade

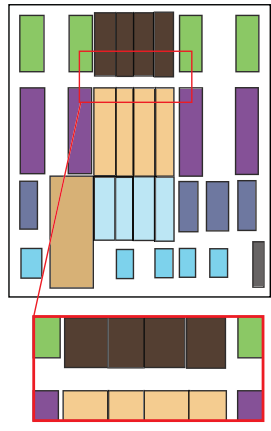

(a) Input layout

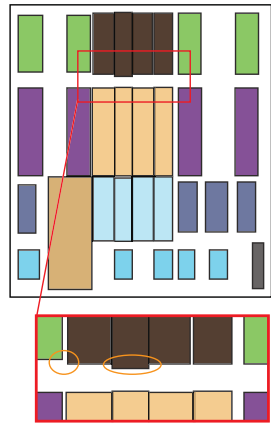

(b) Xu et al. [6]

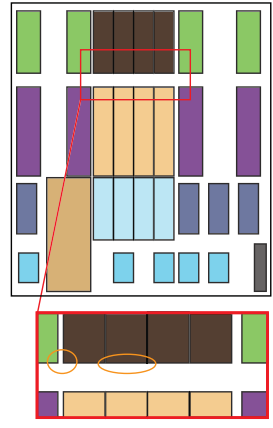

(c) Ours

Fig. 9 A comparison of $\mathrm{Xu}$ et al. [6](b) and our method (c). The yellow circles indicate the differences. 515 off between precision and recall.

516 Applications. Our method is designed for general 2D 517 layouts. One application is the regularization of digitized ${ }_{519}$ plication is the beautification of user drawn layouts, e.g., 518 layouts, e.g., facade layouts shown in Fig. 6. Another ap-520 slide design (see Fig. 7), poster design, and other graphical 


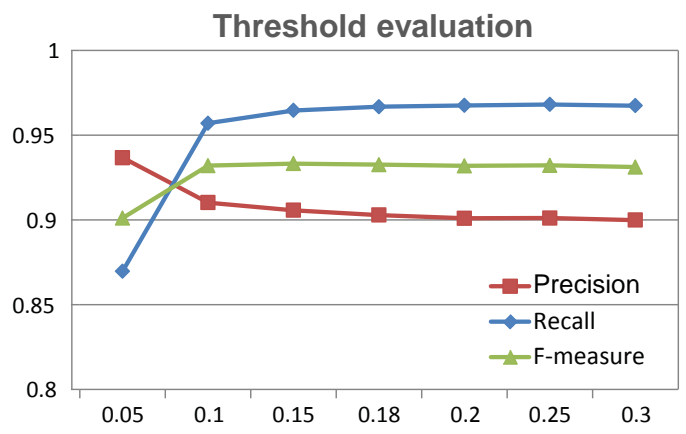

531 constraint. The same arc length constraint enforces a con532 stant arc length along a curve between two adjacent points 533 that are sampled on this curve. In Fig. 13(a), we show a set of 534 markers (the yellow squares) that are placed along the road 535 centerline (in the yellow color). We can see that some adja536 cent markers exhibit the same arc-length constraint. Directly 537 fulfilling this constraint is difficult, considering that we do 538 not know the curve function. We construct a map from a 539 parameter vector to the points by a B-spline interpolation 540 with chord length parameterization. Thus every parameter 541 corresponds to a point, and the interval between two param542 eters is equivalent to the chord length of two adjacent points.

Fig. 10 The robustness of our method with respect to the ${ }_{543}$ Then, we achieve the same arc-length by accomplishing threshold $t_{a}$. We show the change in average precision, ${ }_{544}$ the same spacing constraint on the parametric vectors. In recall, and F-measure for the alignment threshold uniformly ${ }_{545}$ Fig. 13(c), we show the result of this regularization. Another sampled in the range $[0.05,0.3]$.

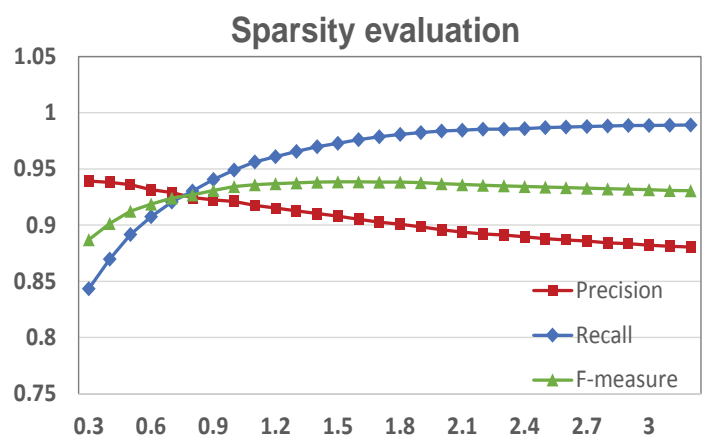

Fig. 11 The robustness of our method with respect to the sparsity term $w_{a}$.

${ }_{521}$ designs (see Fig. 5).

522 Extensions. Our current implementation is developed for 523 axis-aligned layouts, but we can extend our framework to ${ }^{54}$ 524 consider more types of constraints and elements enclosed in ${ }^{54}$ 525 oriented bounding boxes. In Fig. 12, the elements are dis-55 526 tributed on circles. For this example, we introduce two new ${ }^{55}$ 527 types of constraints that consider spacing and alignment in ${ }^{55}$ 528 this radial layouts. Our algorithm can be directly used for 529 these constraints using polar coordinate system.

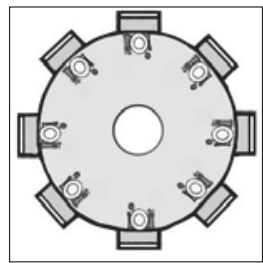

(a) Initial design

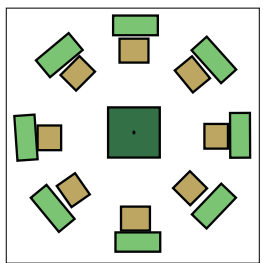

(b) Initial layout

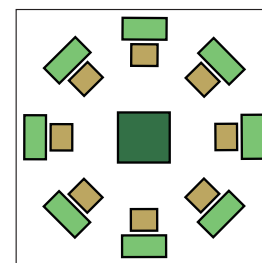

(c) Regularized layout564 and it is unclear how our framework would perform if we

Fig. 12 An extension of our algorithm. In this example, ${ }_{566}$ constraint types. We consider this a very interesting avenue elements (i.e., chairs or the tableware) are expected to be ${ }_{567}$ of future work. Further, we also plan to involve the user in placed along concentric circles with same included angles. ${ }_{568}$ the layout optimization stage to provide more control over The black dot indicates the center of circles. (c) shows the ${ }_{569}$ the regularization process.

result of our algorithm applied to this case by using a simple coordinate system conversion (from the Cartesian coordinate system to the polar coordinate system).

\section{CONCLUSIONS}

571 In this paper, we have presented an optimization-based ap-

Another type of useful constraint is the same arc-length 572 proach for regularizing general layouts. Our method takes 

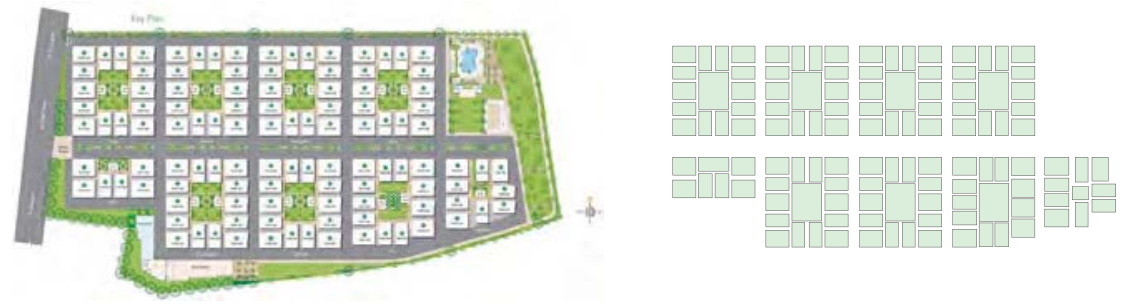

(a) Input design

(b) Regularized result of the 3rd level

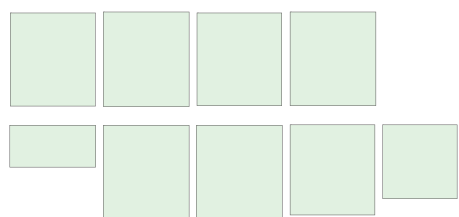

(c) The first level of the input layout

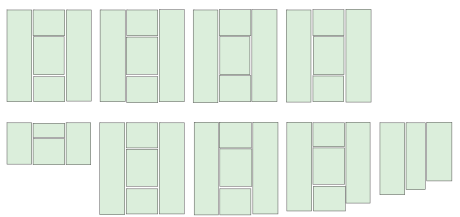

(d) The second level of the input layout

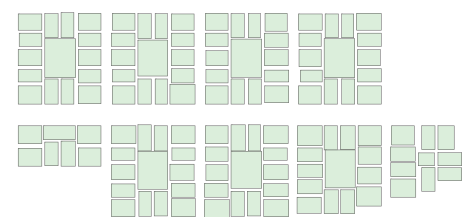

(e) The third level of the input layout

Fig. 14 The regularization of a hierarchical layout. The second row shows the hierarchy from top to down, which is marked by the user. We only use the marked hierarchy to define the group information of lower level layouts.

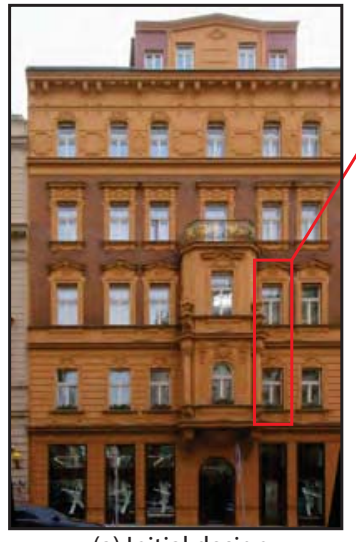

(a) Initial design

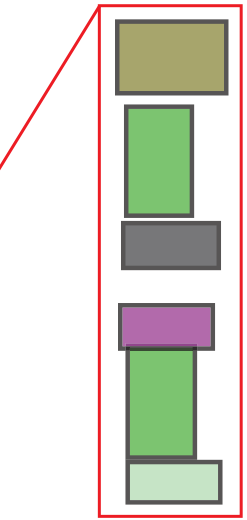

(b) Initial layout

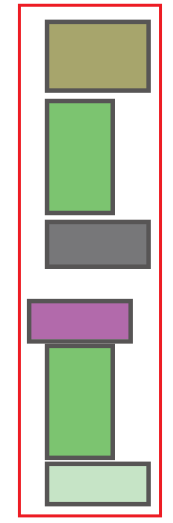

(c) Regularized layout

Fig. 15 A failure case of our algorithm. In this example, the602 user marks a wrong left edge of the ornaments below the ${ }^{603}$ windows in the highlighted region due to occlusions caused ${ }_{605}^{604}$ by perspective projection. Semantic prior information (e.g., 6006 an ornament and window are more likely to be center607 [6] aligned) is necessary to correct this error.

\section{8}

573 as input a general layout represented as a set of labeled rect-612 [8] 574 angles, and detects regularity constraints based on a linear ${ }^{613}$ 575 integer programming formulation. The layout is regularized ${ }_{615}^{614}$ 576 by minimizing the deformation of the initial layout while 616 [9] 577 respecting the detected constraints. We have evaluated our 617 578 method on various input layouts. Experimental results show ${ }^{618}$ 579 that our method enforces the regularities in the layout, and ${ }_{620}^{619}$ 580 is superior to alternative approaches in the literature. We621 [ 581 have also shown the usefulness of our method for various ${ }^{622}$ 582 applications.

\section{${ }_{583}$ ACKNOWLedgmentS}

${ }_{584}$ We would like to thank the reviewers for their helpful ${ }^{628}$ 585 comments, and the authors of [6] for making their software ${ }_{630}^{629}$ ${ }_{586}$ public available and their help for the comparison. This 631
${ }_{587}$ work was supported by the KAUST Visual Computing 588 Center, the National Natural Science Foundation of China $589(61372168,61331018,61271431$, and 61272327), and the U.S. 590 National Science Foundation.

\section{References}

2 [1] F. Wu, D.-M. Yan, W. Dong, X. Zhang, and P. Wonka, "Inverse procedural modeling of facade layouts," ACM TOG (SIGGRAPH), vol. 33, no. 4, pp. 121:1-121:10, Jul. 2014.

[2] C. Wu, J.-M. Frahm, and M. Pollefeys, "Detecting large repetitive structures with salient boundaries," in ECCV. Springer, 2010, pp. 142-155.

[3] M. Pauly, N. J. Mitra, J. Wallner, H. Pottmann, and L. J. Guibas, "Discovering structural regularity in 3d geometry," in ACM Transactions on Graphics (TOG), vol. 27, no. 3. ACM, 2008, p. 43.

[4] N. J. Mitra, A. Bronstein, and M. Bronstein, "Intrinsic regularity detection in 3d geometry," in Computer Vision-ECCV 2010. Springer, 2010, pp. 398-410.

[5] T. Pavlidis and C. J. Van Wyk, "An automatic beautifier for drawings and illustrations," Computer Graphics (Proc. SIGGRAPH), vol. 19, no. 3, pp. 225-234, Jul. 1985.

P. Xu, H. Fu, T. Igarashi, and C.-L. Tai, "Global beautification of layouts with interactive ambiguity resolution," in UIST '14, 2014.

P. Musialski, P. Wonka, D. G. Aliaga, M. Wimmer, L. van Gool, and W. Purgathofer, "A survey of urban reconstruction," Computer Graphics Forum, vol. 32, no. 6, pp. 146-177, 2013.

8] D. Dai, M. Prasad, G. Schmitt, and L. Van Gool, "Learning domain knowledge for facade labelling," in Proceedings of the 12th European Conference on Computer Vision - Volume Part I, ser. ECCV'12, 2012, pp. $710-723$.

] M. Park, K. Brocklehurst, R. T. Collins, and Y. Liu, “Translationsymmetry-based perceptual grouping with applications to urban scenes," in ACCV, 2011, pp. 329-342.

10] C. Wu, J.-M. Frahm, and M. Pollefeys, "Repetition-based dense single-view reconstruction," in CVPR, 2011, pp. 3113-3120.

11] R. H., K. U., T. W., D. M., H. S., and B. H. Fellner D., "Irregular lattices for complex shape grammar facade parsing," in CVPR, 2012, p. 16401647.

624 [12] C. Yang, T. Han, L. Quan, and C.-L. Tai, "Parsing facade with rankone approximation," in CVPR, 2012, pp. 1720-1727.

[13] O. Teboul, I. Kokkinos, L. Simon, P. Koutsourakis, and N. Paragios, "Parsing facades with shape grammars and reinforcement learning," IEEE PAMI, vol. 35, no. 7, pp. 1744-1756, 2013.

[14] C.-H. Shen, S.-S. Huang, H. Fu, and S.-M. Hu, "Adaptive partitioning of urban facades," ACM Transactions on Graphics (Proceedings of ACM SIGGRAPH ASIA 2011), vol. 30, no. 6, pp. 184:1-184:9, 2011. 
632 [15] P. Musialski, M. Wimmer, and P. Wonka, "Interactive coherence-698 633 based facade modeling," Comp. Graph. Forum, vol. 31, no. 23, pp.699 634 661-670, May 2012.

635 [16] H. Zhang, K. Xu, W. Jiang, J. Lin, D. Cohen-Or, and B. Chen, “Lay-701 636 ered analysis of irregular facades via symmetry maximization," 702 637 ACM Transactions on Graphics (Proceedings of ACM SIGGRAPH703 638 2013), vol. 32, no. 4, pp. 121:1-121:10, 2013

639 [17] N. J. Mitra, L. Guibas, and M. Pauly, "Partial and approximate 705 640 symmetry detection for $3 \mathrm{~d}$ geometry," ACM Transactions on Graph641 ics (SIGGRAPH), vol. 25, no. 3, pp. 560-568, 2006.

642 [18] M. Pauly, N. J. Mitra, J. Wallner, H. Pottmann, and L. Guibas, “Dis643 covering structural regularity in 3D geometry," ACM Transactions 644 on Graphics, vol. 27, no. 3, pp. \#43, 1-11, 2008.

645 [19] A. Tevs, Q. Huang, M. Wand, H.-P. Seidel, and L. Guibas, "Relating 646 shapes via geometric symmetries and regularities," ACM TOG, $647 \quad$ vol. 33, no. 4, pp. 119:1-119:12, Jul. 2014.

648 [20] Y. Li, X. Wu, Y. Chrysanthou, A. Sharf, D. Cohen-Or, and N. J. Mi- ${ }^{707}$ 649 tra, "Globfit: Consistently fitting primitives by discovering global ${ }^{708}$

692 [35] GLPK, http://www.gnu.org/software/glpk/.

693 [36] M. Dang, D. Ceylan, B. Neubert, and M. Pauly, "Safe: Structure694 aware facade editing," vol. 33, no. 2, pp. 83-93, 2014.

695 [37] F. Bao, M. Schwarz, and P. Wonka, "Procedural facade variations 696 from a single layout," ACM Trans. Graph., vol. 32, no. 1, pp. 8:1-

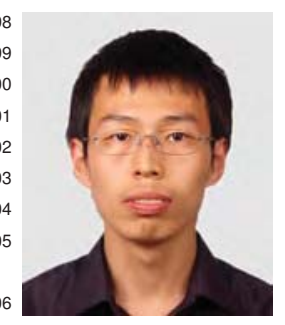

Haiyong Jiang is a Ph.D. student at the National Laboratory of Pattern Recognition of the Institute of Automation, Chinese Academy of Sciences (CAS). He received his Bachelor's degrees from University of Science and Technology Beijing in 2012. His research interests lie in computer graphics and computer vision.

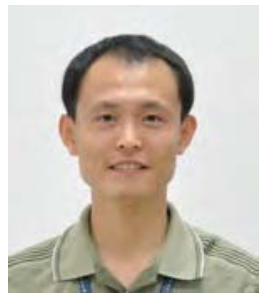

Liangliang Nan received his bachelor's degree from Nanjing University of Aeronautics and Astronautics (NUAA), in 2003 and the Ph.D. degree from Shenyang Institute of Automation (SIA), Chinese Academy of Sciences in 2009. He currently works as a research scientist at King Abdullah University of Science and Technology (KAUST). His research interests lie in the fields of computer graphics, computer vision, and human-computer interaction.

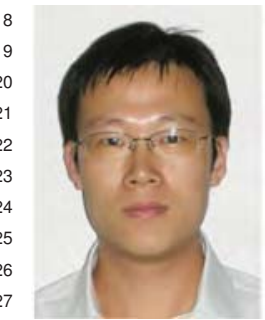

Dong-Ming Yan is a research scientist at King Abdullah University of Science and Technology (KAUST), and he is an associate professor at the National Laboratory of Pattern Recognition of the Institute of Automation, Chinese Academy of Sciences (CAS). He received his Ph.D. from Hong Kong University in 2010 and his Master's and Bachelor's degrees from Tsinghua University in 2005 and 2002, respectively. His research interests include computer graphics, geometric processing and visualization.

Weiming Dong is an associate professor in the Sino-French Laboratory (LIAMA) and National Laboratory of Pattern Recognition (NLPR) at Institute of Automation, Chinese Academy of Sciences. He received his BSc and MSc degrees in computer science in 2001 and 2004, both from Tsinghua University, P. R. China. He received his $\mathrm{PhD}$ in computer science from the University of Henri Poincaré Nancy 1, France, in 2007. His research interests include image synthesis and image analysis. Weiming Dong is a member of $740 \mathrm{ACM}$ and IEEE.

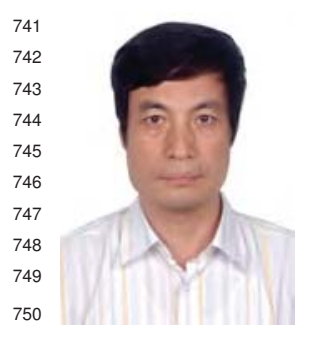

Xiaopeng Zhang is a professor at the National Laboratory of Pattern Recognition of the Institute of Automation, Chinese Academy of Sciences (CAS). He received his Ph.D. degree in Computer Science from the Institute of Software, CAS in 1999. He received the National Scientific and Technological Progress Prize (second class) in 2004. His main research interests include computer graphics and image processing. 
This article has been accepted for publication in a future issue of this journal, but has not been fully edited. Content may change prior to final publication. Citation information: DOI

10.1109/TVCG.2015.2480059, IEEE Transactions on Visualization and Computer Graphics

JOURNAL OF TVCG

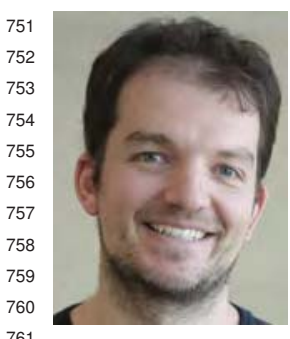

Peter Wonka received his Ph.D. degree in computer science and M.S. degree in urban planning from the Technical University of Vienna, Vienna, Austria, in 2001 and 2002, respectively. He was a postdoctoral researcher with the Georgia Institute of Technology, Atlanta, GA, USA, for two years. He is currently a professor with the Computer, Electrical and Mathematical Sciences and Engineering Division, King Abdullah University of Science and Technology, Thuwal, Saudi Arabia, and also an associate professor with Arizona 762 State University, Tempe, USA. His research interests include topics 763 in computer graphics, visualization, computer vision, remote sensing, 764 image processing, and machine learning. 